After Silence 



\section{After Silence}

A History of AIDS through

Its Images

AVRAM FINKELSTEIN

甲 
University of California Press, one of the most distinguished university presses in the United States, enriches lives around the world by advancing scholarship in the humanities, social sciences, and natural sciences. Its activities are supported by the UC Press Foundation and by philanthropic contributions from individuals and institutions. For more information, visit www.ucpress.edu.

University of California Press

Oakland, California

(ㄷ) 2018 by Avram Finkelstein

Library of Congress Cataloging-in-Publication Data

Names: Finkelstein, Avram, 1952- author.

Title: After silence : a history of AIDS through its images / Avram Finkelstein.

Description: Oakland, California : University of California Press, [2018] | Includes index.

Identifiers: LCCN 2017O29451| ISBN 9780520295148

(cloth : alk. paper) | ISBN 9780520968028 (ebook)

Subjects: LCSH: AIDS (Disease) and the arts-United

States. | AIDS activists-United States. |

Finkelstein, Avram, 1952- $\mid$ Silence = Death

Project (Artists' collective) | Gran Fury (Artists'

collective) | ACT U P (Organization) | AIDS

(Disease)-United States-History.

Classification: LCC NX180.A36 F56 2018 | DDC

701/.03-dc23

LC record available at

https://lccn.loc.gov/2017029451

$\begin{array}{llllllllll}27 & 26 & 25 & 24 & 23 & 22 & 21 & 20 & 19 & 18\end{array}$

$\begin{array}{llllllllll}10 & 9 & 8 & 7 & 6 & 5 & 4 & 3 & 2 & 1\end{array}$ 